\title{
Changes in the concentration of fructose in the blood of piglets of different ages after doses of fructose, fructose plus glucose, and sucrose
}

\author{
BY P. H. BIRD* AND P. E. HARTMANN \\ Department of Biochemistry, University of Western Australia, Nedlands WA 6009, Australia
}

(Received 16 August 1994 - Revised 7 November 1995 - Accepted 3 January 1996)

\begin{abstract}
We investigated the hydrolysis of sucrose in the small intestine and the subsequent absorption and metabolism of fructose in sucking piglets by measuring temporal changes in the concentration of fructose in the plasma following the administration of physiological amounts of these carbohydrates. Calculations of the area under the curve for fructose in the plasma showed that there was no age limit to the piglets' ability to absorb fructose. However, there was a limit to the amount of fructose that the younger piglets could get from a dose of sucrose. Indeed, we demonstrated that there was a positive linear correlation between a piglet's capacity to hydrolyse sucrose and the age of the piglet up to $15 \mathrm{~d}$ of age ( $\mathrm{r} 0.98)$. The half-life for fructose was $495,103,38,49$ and $28 \mathrm{~min}$ in 2-, 5-, 7-, 10- and 15-d-old piglets respectively and, thus, there was only limited utilization of fructose in the younger piglets. However, there were 13.0- and 1.4-fold increases in the elimination rate of fructose from the plasma of piglets from 2 to $7 \mathrm{~d}$ and from 7 to $15 \mathrm{~d}$ respectively, consistent with the reported increase in the deposition of fat in piglets of a similar age range. Hence, the effective metabolism of fructose may be partially dependent on the amount of adipose tissue present and the phosphorylation of this monosaccharide by hexokinase $(E C$ 2.7.1.1) in this tissue.
\end{abstract}

Sucrose: Fructose: Piglet

The concentration of fructose in the blood of the newborn piglet is relatively high (about $3.8 \mathrm{~mm}$ ) at birth, but then decreases rapidly to $1.5 \mathrm{mM}$ after $8 \mathrm{~h}$ and reaches undetectable concentrations by $2 \mathrm{~d}$ of age (Curtis et al. 1966). Since the disappearance of fructose can be accounted for by loss in the urine, it has been concluded that this monosaccharide is not metabolized to any significant extent and, therefore, it can only have a minor role as an energy source for the newborn piglet (Aherne et al. $1969 \mathrm{~b}$ ). Several workers have found very little sucrase (EC 3.2.1.48) activity in the small intestine of newborn pigs, some activity at 1 week and appreciable amounts of activity at 2 weeks of age (Bailey et al. 1956; Walker, 1959; Dahlqvist, 1961; Hartman et al. 1961; Manners \& Stevens, 1972; James et al. 1987). Furthermore, previous studies have shown a marked change in the concentration of fructose in the blood between 2 and 17-d-old piglets after a dose of either fructose (Kidder et al. 1963; Aherne et al. 1969a) or sucrose (Kidder et al. 1963).

Calculations based on the results of other investigations demonstrate that piglets typically ingest between $1.18 \mathrm{~g}$ (Barber et al. 1955) and $1.7 \mathrm{~g}$ of lactose (Atwood et al. 1990) each time they suckle the sow. The smallest doses of sugar given to piglets in previous in vivo investigations into the metabolism of fructose and sucrose contained about $2.5 \mathrm{~g}$

* Present address: CSIRO, Division of Wildlife and Ecology, PO Box 84, Lyneham, ACT 2602, Australia. 
fructose, $5 \mathrm{~g}$ sucrose (Kidder et al. 1963) and $2.0 \mathrm{~g}$ fructose (Aherne et al. 1969a). Therefore, these doses of fructose $(2.5 \mathrm{~g})$ and sucrose $(5 \mathrm{~g})$ represent an unusually high intake of simple carbohydrate for sucking piglets.

We report on our investigations of the metabolism of fructose and sucrose by piglets (from 2 to $15 \mathrm{~d}$ of age) after the ingestion of these sugars in amounts comparable with the carbohydrate intake from a natural sucking. Furthermore, the hypothesis that the capacity of piglets to digest physiological amounts of sucrose and metabolize fructose increases with age was tested.

\section{MATERIALS AND METHODS}

Animals

Piglets ( $n 6 ; 10 \mathrm{~d}$ old), with a mean body weight of 3.4 (SD $0 \cdot 3) \mathrm{kg}$, were used to investigate the basal concentration of fructose. A second group of 10 -d-old piglets $(n 3)$, with a mean body weight of 3.5 (SD 0.5 ) $\mathrm{kg}$, was used to investigate the changes in the concentration of fructose following an intravenous dose of fructose. Groups of 2-, 5-, 7-, 10- and 15-d-old piglets, with mean body weights of 1.9 (SD $0.4 ; n 6), 2 \cdot 2$ (SD $0.2 ; n 6), 2.9(\operatorname{SD~} 0.3 ; n 5)$, 3.5 (SD $0.4 ; n 5$ ) and 5.0 (SD $0.6 ; n 6) \mathrm{kg}$ respectively, were treated with oral doses of fructose plus glucose. Further groups of 2-, 5-, 7-, 10- and 15-d-old piglets, with mean body weights of $1.9(\operatorname{SD~} 0.1 ; n 6), 2.6(\operatorname{SD~} 0.2 ; n 6), 2.9(\operatorname{SD~} 0.4 ; n 5), 3.6(\operatorname{SD~} 0.3 ; n 6)$ and $4.5(\operatorname{SD~} 0.6$; $n 6) \mathrm{kg}$, were treated with oral doses of sucrose. All piglets were healthy, selected at random and separated from their sow by partitioning the farrowing crate.

\section{Treatments and blood sampling}

All piglets were separated from their sow for at least $1 \mathrm{~h}$ before treatments. The first group of piglets received no prior administration of either an intravenous or an oral dose of any solution. The remaining piglets received either an intravenous or an oral dose. The administration time for each dose was between 10 and $30 \mathrm{~s}$ for the intravenous doses and between 0.5 and 2 min for the oral doses. Zero time was taken as the time when half the volume of the solution had been administered. The ear veins of the piglet were pricked with finger lancets (Auto Lancets, Miles Laboratories, IN, USA) and blood (50-60 $\mu \mathrm{l}$ ) was collected in heparinized capillary tubes (Chase Instruments Corp., Glens Falls, NY, USA) and immediately placed on ice. When piglets were administered an intravenous dose into the ear vein, blood was collected from the veins in the opposite ear of the piglet. Blood samples were collected just before each dose and at 3-10 min intervals. Each capillary was sealed at one end with a micro-packed cell volume tube closure (Critocaps ${ }^{\mathbb{R}}$, Monoject Scientific, St Louis, MO, USA) and the plasma separated from the erythrocytes by centrifugation on a Clements bench centrifuge (Westral Scientific, Australia) at $8000 \mathrm{~g}$ for $5 \mathrm{~min}$. The tubes were stored at $-20^{\circ}$ until assayed.

\section{Dosing regimes}

Treatment ND: no prior administration of either an intravenous or an oral dose of any solution.

Treatment IV: an intravenous dose of a $250 \mathrm{~g} / 1$ fructose solution ( $60 \mathrm{mg}$ fructose $\mathrm{kg}$ body weight) was injected into the ear vein with a single injection $(10 \mathrm{~d}$ old $)$.

Treatment FRU + GLU: a solution $(15 \mathrm{ml})$ containing $0.675 \mathrm{~g}$ fructose plus $0.675 \mathrm{~g}$ glucose was administered orally.

Treatment SUC: a solution (15 ml) containing $1.35 \mathrm{~g}$ sucrose was administered orally. 


\section{Biochemical analysis}

The plasma (20 $\mu \mathrm{l}$ ) was deproteinized with $200 \mu \mathrm{l} \mathrm{0.6} \mathrm{M-perchloric} \mathrm{acid} \mathrm{(Arthur} \mathrm{et} \mathrm{al.} \mathrm{1989)}$ and the concentration of fructose was determined (Guppy et al. 1990).

\section{Data analysis}

The concentrations of fructose in the plasma of the piglets that were given either the intravenous or oral doses of fructose were plotted against time. The half-life of fructose in the plasma and the area under the curve (AUC) were calculated (Bird \& Hartmann, 1994). The time taken for the concentration of fructose in the plasma to reach half the initial concentration following an intravenous dose of fructose (half-life) during a typical sucking interval $\left(1 \mathrm{~h}\right.$ ) was calculated from the elimination slope given that $\mathrm{C}=\mathrm{C}_{0}{ }^{-\mathrm{kt}}$ (Bird \& Hartmann, 1994). We found that following the dosing of piglets with the same amount of fructose, the peak concentration in the plasma decreased as the age of the piglets increased (see Fig. 3). Since piglets of different body weights were administered the same amounts of either fructose plus glucose or sucrose, the heavier piglets distributed the absorbed fructose in a larger volume of plasma and, therefore, the calculated fructose AUC was adjusted by a dilution factor so that the index of the total amount of fructose in the peripheral plasma of piglets with different plasma volumes could be compared (Bird \& Hartmann, 1994). Following the dose of fructose plus glucose (treatment FRU + GLU), the results showed that the concentration of fructose in the plasma did not further increase after $40 \mathrm{~min}$ (see Fig. 3). Therefore, this time point of $\mathbf{4 0} \mathrm{min}$ after the administration of the dose was selected as the cut-off time to calculate the adjusted fructose $A U C$, which represents the main absorptive time for a dose of fructose.

The relative digestive capacity for sucrose was obtained by using a similar method to that previously described for lactose (Bird et al. 1995). In order to approximate the normal sucking frequency of the piglets in our previous studies investigating the hydrolysis of lactose (Bird \& Hartmann, 1994), the doses of galactose plus glucose were administered after the piglets were separated for $60 \mathrm{~min}$ and the lactose was administered 90 min later. However, preliminary investigations demonstrated that the disappearance rate of fructose from the peripheral blood of younger piglets was not sufficiently fast to clear all this monosaccharide within a normal sucking interval. Since we were investigating piglets of a particular age, but were not able to dose the same piglet with both fructose plus glucose and sucrose at an interval which approximated the normal sucking frequency of $1 \mathrm{~h}$ (Fraser, 1980), different piglets within each age group were chosen for treatments FRU + GLU and SUC. Therefore, we calculated the relative digestive capacity for sucrose for each age group using the following formula:

$$
\frac{\text { mean adjusted fructose AUC (treatment SUC) }}{\text { mean adjusted fructose AUC (treatment FRU }+ \text { GLU) }} \times 100 \text {. }
$$

\section{Statistical analysis}

Significant differences in the adjusted fructose AUC were tested with two-way ANOVA. The relationship between the relative digestive capacity and the age of the piglet was determined by regression analysis. Results are expressed as mean and either the standard deviation (SD), standard error of the mean (SEM), or the standard error of the difference between the means (SED: pooled estimate of the error). Statistical analysis was determined using StatView, SE + Graphics TM II, v1.03, Abacus Concepts Inc. Berkeley, USA. 


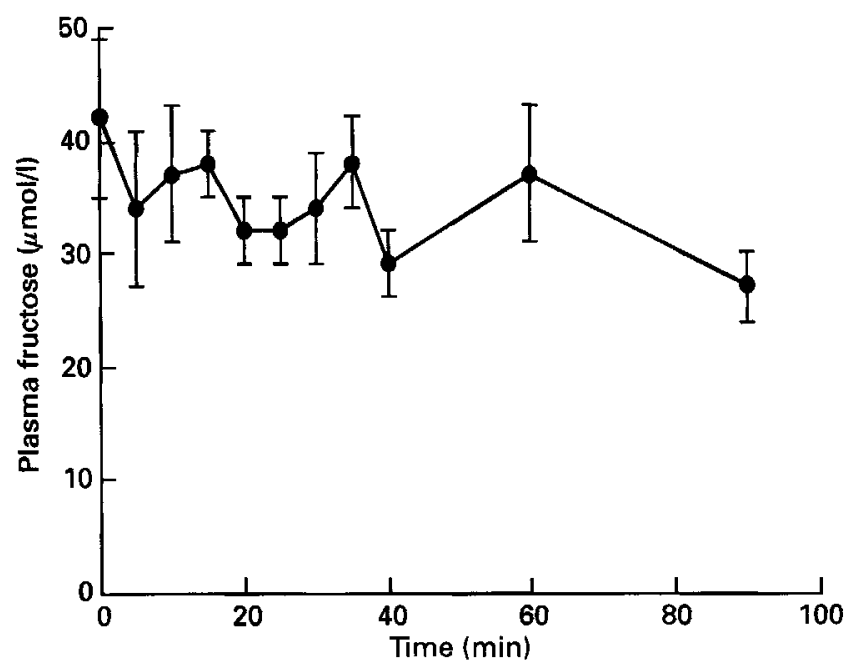

Fig. 1. The fasting concentration of fructose in the plasma $v$. time in 10-d-old piglets during handling and sampling procedures (treatment ND). Values are means for six piglets, with their standard errors represented by vertical bars.

\section{RESULTS}

\section{Basal concentration and intravenous doses}

The mean basal concentrations of fructose in the 10-d-old piglets $(n 6)$ for treatment ND are shown in Fig. 1. The mean concentration of fructose in the plasma of the piglets during this time was 34.6 (SD 11.37) $\mu \mathrm{mol} / 1$.

The clearance of fructose following an intravenous dose of fructose (treatment IV) into 10-d-old piglets is shown in Fig. 2. The half-life of fructose in the plasma following treatment IV was calculated to be $50 \mathrm{~min}, \log y=2.93-0.006 x, r 0.97$ (piglet 1), $25 \mathrm{~min}$, $\log y=2.90-0.012 x, r 0.96$ (piglet 2) and $27 \mathrm{~min}, \log y=2.98-0.011 x, r 0.91$ (piglet 3).

\section{Oral doses of sugars}

The concentration of fructose in the peripheral plasma of piglets of different age groups after a dose of either fructose plus glucose (treatment FRU + GLU) or sucrose (treatment SUC) is presented in Fig. 3. The concentrations of fructose in the plasma of 2-, 5-, 7-, 10and 15-d-old piglets, after $1 \mathrm{~h}$ of separation from the sow, were 106.5 (SD 88), 58.4 (SD 21 ), 98.6 (SD 84), 36.6 (SD 5.6) and 28.8 (SD 16.7) $\mu \mathrm{mol} / 1$ respectively. There was a significant increase $(P<0.05)$ in the concentration of fructose in the plasma after 10 min for the 2-, 5-, 7- and 15-d-old piglets and after $15 \mathrm{~min}$ for the 10 -d-old piglets following treatment FRU + GLU (Fig. 3). Furthermore, there was a significant increase $(P<0.05)$ in the concentration of fructose in the plasma after $25 \mathrm{~min}$ for the 2-and 10-d-old piglets, $20 \mathrm{~min}$ for the 5-d-old piglets, $10 \mathrm{~min}$ for the 7 -d-old piglets and $5 \mathrm{~min}$ for the 15-d-old piglets following treatment SUC (Fig. 3).

Whereas the peak concentrations of fructose in the plasma following treatment FRU + GLU were significantly different $(P<0.05)$ to those following treatment SUC for the 2-, 5- and 7-d-old piglets (Figs. 3(a), (b) and (c)), they were not significantly different for the 10- and 15-d-old piglets (Figs. 3(d) and (e)). Regression equations for the logarithm of the post-absorptive decline of plasma fructose $v$. time, following treatment FRU + GLU (Fig. 3), were: $\log y=3.21-0.0006 x, r 0.93 ; \log y=3.34-0.0029 x, r 0.96 ; \log y=$ $3.36-0.0089 x, r 0.97 ; \log y=3.03-0.0061 x, r 0.98 ; \log y=3.06-0.011 x, r 0.99$ for the 2-, 

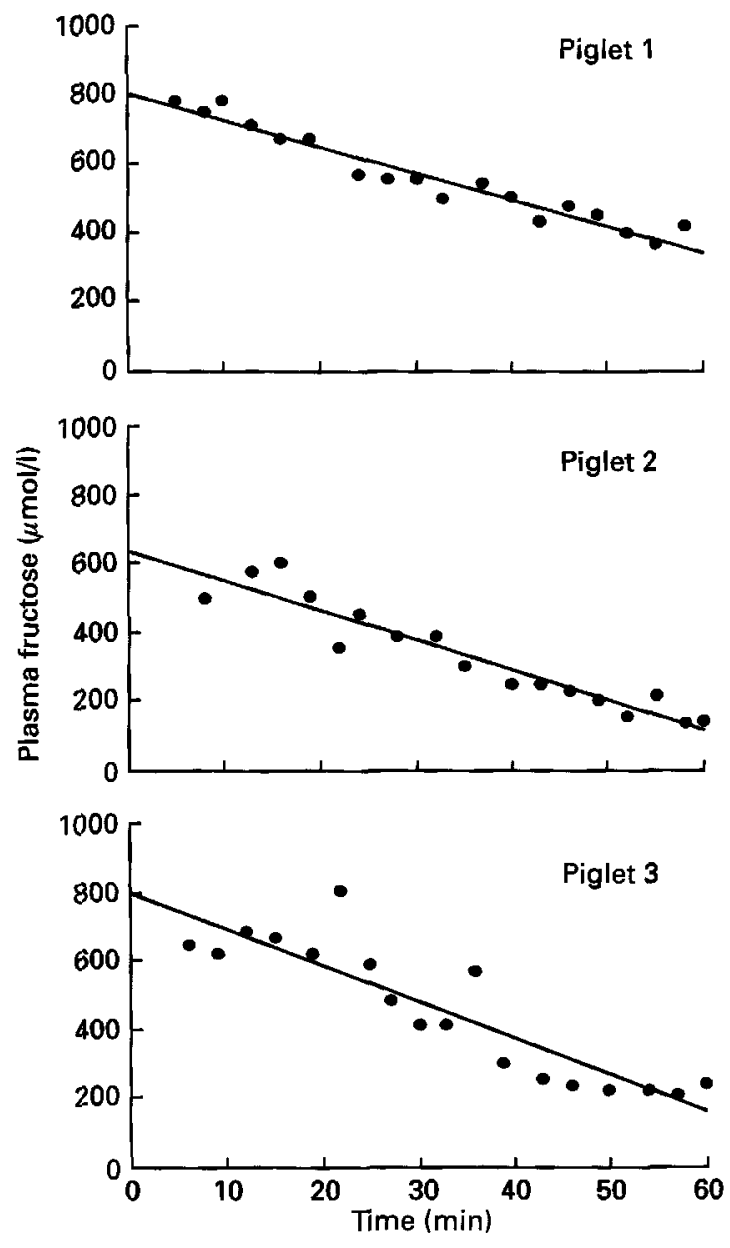

Fig. 2. The concentrations of fructose in the plasma $v$. time in three 10-d-old piglets following an intravenous dose (60 $\mathrm{mg} / \mathrm{kg}$ body weight) of fructose $(250 \mathrm{~g} / \mathrm{l})$ (treatment IV).

5-, 7-, 10- and 15-d-old piglets respectively. The half-life of fructose in the plasma was calculated and found to be $495,103,38,49$ and $28 \mathrm{~min}$ for the 2-, 5-, 7-, 10- and 15-d-old piglets respectively.

The adjusted fructose AUC following treatment FRU + GLU was significantly higher than the adjusted fructose AUC following treatment SUC for the 2-, 5- and 7-d-old piglets. There were no significant differences in the adjusted fructose AUC between the different age groups following treatment FRU + GLU. However, there was a significant difference in the adjusted fructose AUC between the 2-d-old and the older piglets following treatment SUC. Furthermore, there was a significant difference in the adjusted fructose AUC between the 15-d-old and the 5-, 7- and 10-d-old piglets following treatment SUC (Table 1). Transforming the data to give the $\log _{10}$ of the adjusted fructose AUC gave the same statistical results. The piglets' relative digestive capacity for sucrose in each of the different age groups is shown in Table 1 . There was a significant linear correlation $(r 0.98, n 5)$ between the relative digestive capacity for sucrose and the age of the piglet up to $15 \mathrm{~d}$ of age. 


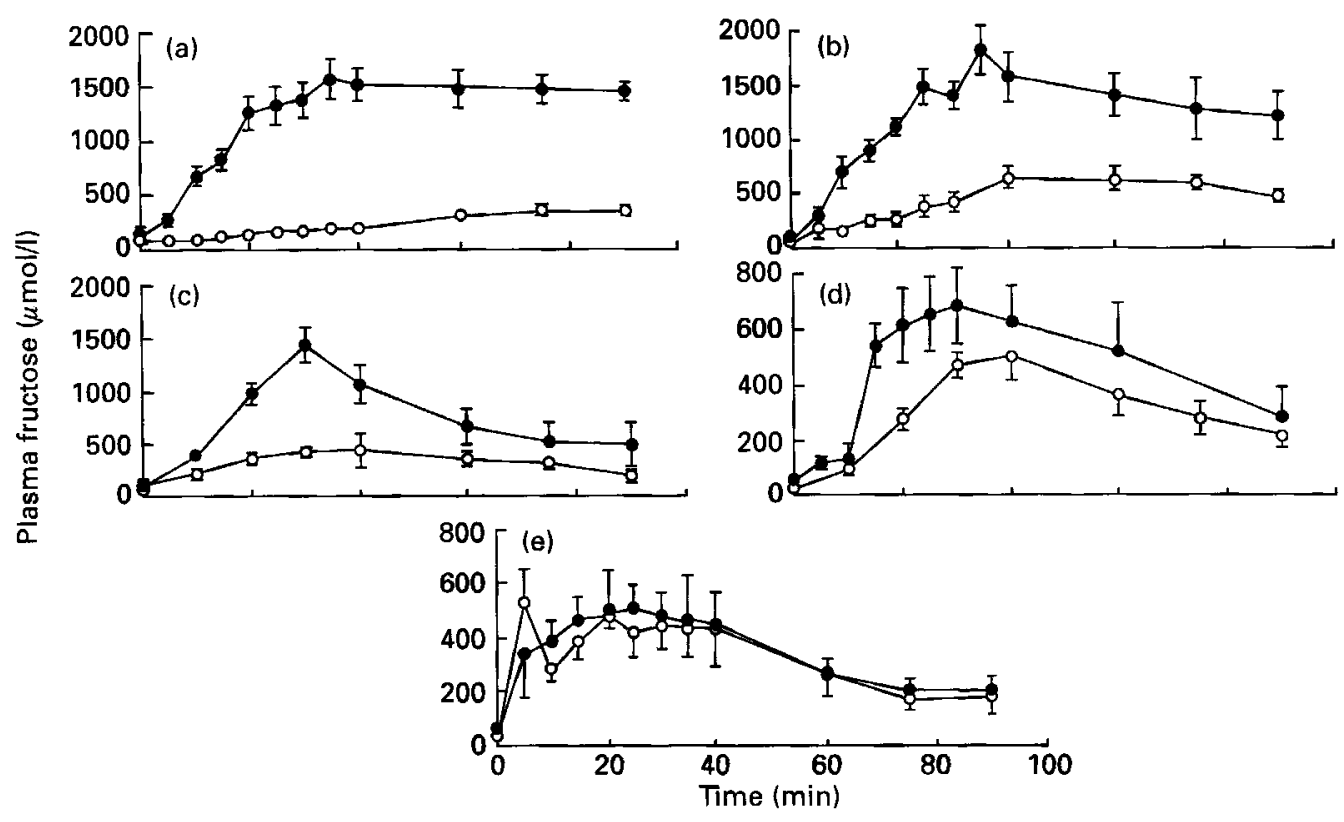

Fig. 3. The concentrations of fructose in the plasma $v$. time in piglets following oral doses of either sucrose (O, treatment SUC) or fructose plus glucose (9, treatment FRU +GLU). (a) Piglets $2 \mathrm{~d}$ old, $n 6$; (b) $5 \mathrm{~d}$ old, $n 6$; (c) $7 \mathrm{~d}$ old, $n 5$; (d) $10 \mathrm{~d}$ old, $n 6$; (e) $15 \mathrm{~d}$ old, $n 6$. Values are means with their standard errors represented by vertical bars.

Table 1. Mean adjusted fructose areas under the curve $(A U C)$ and the $\log _{10}$ of the adjusted fructose $A U C$ after doses of sucrose $(S U C)$ or fructose plus glucose $(F R U+G L U)$ in piglets of different ages*

\begin{tabular}{|c|c|c|c|c|c|c|c|}
\hline \multirow[b]{2}{*}{ Age (d) } & \multicolumn{3}{|c|}{ Treatment SUC } & \multicolumn{3}{|c|}{ Treatment FRU + GLU } & \multirow{2}{*}{$\begin{array}{l}\text { Relative digestive } \\
\text { capacity }(\%) \dagger\end{array}$} \\
\hline & $n$ & AUC & $\log _{10}$ & $n$ & AUC & $\log _{10}$ & \\
\hline 2 & 6 & $1 \cdot 3^{\mathrm{a}}$ & 0.07 & 6 & $8 \cdot 6^{\mathrm{b}}$ & 0.92 & 15 \\
\hline 5 & 6 & $3 \cdot 8^{\mathrm{a}}$ & 0.57 & 6 & $9 \cdot 8^{b}$ & 0.97 & 39 \\
\hline 7 & 5 & $3.9^{\mathrm{a}}$ & 0.57 & 5 & $10 \cdot 4^{b}$ & 0.99 & 38 \\
\hline 10 & 6 & $4 \cdot 1$ & 0.57 & 5 & $7 \cdot 0$ & 0.79 & 59 \\
\hline 15 & 6 & $6 \cdot 8$ & 0.81 & 6 & $7 \cdot 0$ & 0.79 & 97 \\
\hline SED & & 1.46 & 0.08 & & 1.46 & 0.08 & - \\
\hline
\end{tabular}

a, b Mean values within a row with unlike superscript letters were significantly different $(P<0.05$; two-way ANOVA).

* For details of treatments and procedures, see pp. 400-401.

$\dagger$ Calculated from the formula:

$\frac{\text { mean adjusted fructose AUC (treatment SUC) }}{\text { mean adjusted fructose AUC (treatment FRU +GLU) }} \times 100$.

\section{DISCUSSION}

The concentration of fructose in the peripheral plasma of the piglets was not affected by the sampling procedures (treatment ND, Fig. 1), whereas it increased significantly following a dose of fructose (treatment FRU + GLU, Fig. 3). Indeed, the profiles of the changes in the concentration of fructose in the plasma (Fig. 3) were similar to those reported in previous studies for piglets of $2-17 \mathrm{~d}$ of age following larger doses of fructose 
(Kidder et al. 1963; Aherne et al. 1969 a). However, the concentrations of fructose in the plasma reported by these authors were higher and remained elevated for a longer period of time. This difference was a consequence of the smaller amounts of carbohydrate, equivalent to the amount of lactose ingested during a typical sucking, administered in the present study.

Whereas galactose and glucose, the major monosaccharides derived from sow's milk, are absorbed in the small intestine by an active transport system, fructose appears to be absorbed by facilitated diffusion (Hopfer, 1987). Previously, we have shown that the efficiency of galactose absorption is not affected by the age of the sucking piglet (Bird et al. 1995). The current study (Table 1) showed that there was no significant difference in the adjusted fructose AUC over the first 40 min following treatment FRU + GLU in piglets from 2- to 15-d of age. This finding implies that similar amounts of fructose reached the peripheral plasma of the piglets from the different age groups after they had been administered the same amounts of fructose. Therefore, there did not appear to be any age limit to the absorption of $0.675 \mathrm{~g}$ fructose by the small intestine of piglets. However, we demonstrated that the difference in the absorption of fructose after a dose of fructose (treatment FRU + GLU) compared with a dose of sucrose (treatment SUC) was much greater in the 2-, 5- and 7-d-old piglets than in the 10- and 15-d-old piglets. These findings are consistent with those of Kidder et al. (1963) who observed that the mucosa of the 6-d-old piglet had a limited capacity to hydrolyse sucrose compared with that of 17-d-old piglets.

Several studies in piglets have demonstrated that the activity of sucrase is low at birth (Bailey et al. 1956; Walker, 1959; Dahlqvist, 1961; Hartman et al. 1961; Manners \& Stevens, 1972; James et al. 1987). Furthermore, Walker (1959) and Manners \& Stevens (1972) found an almost linear increase in sucrase activity from birth to 3 weeks of age. We calculated the piglet's relative digestive capacity for sucrose (Table 1) and demonstrated that there was a significant linear correlation $(y=2 \cdot 11+6 \cdot 09 x, r 0 \cdot 983, n 5)$ between the relative digestive capacity and age in piglets from $2-15 \mathrm{~d}$ of age. Extrapolation of this linear regression to birth (0-d-old) demonstrated that there was a $1 \%$ relative digestive capacity for sucrose at this time. The findings of Dollar et al. (1957) and Kidder et al. (1963) on the increase in the concentration of reducing sugars and fructose respectively in the blood after larger oral doses of sucrose were consistent with our findings. Furthermore, our functional measurement of relative digestive capacity is in agreement with observations by Bailey et al. (1956) and Walker (1959) on the increase in the activity of sucrase in piglet mucosa with age, and by Johnson (1949) and Becker et al. (1954) that piglets under 1 week of age receiving diets based on very high amounts of sucrose have relatively poor growth performance.

The first step of fructose metabolism is the ATP-dependent phosphorylation to fructose1-phosphate by fructokinase $(E C 2.7 .1 .4)$ located almost exclusively in the liver (Shafrir, 1985). However, hexokinase (EC 2.7.1.1) also actively phosphorylates fructose in vitro. The half-life of fructose in the blood, calculated from the FRU + GLU treatment, decreased from 495, 103, 38, 49 to 29 min from 2, 5, 7, 10 to 15 d respectively, and this was consistent with the report by Aherne et al. $(1969 a)$ on the higher activity of fructokinase found in the liver of 9 -d-old $(3.66 \mu \mathrm{mol} / \mathrm{min}$ per $\mathrm{g}$ tissue) compared with 6 -d-old piglets $(2.68 \mu \mathrm{mol} / \mathrm{min}$ per $\mathrm{g}$ tissue). Since the excretion of ingested fructose decreased from a major to a minor component of fructose clearance from day 0 to $6 \mathrm{~d}$ postpartum in piglets (Aherne et al. 1969 a), the metabolism of fructose must have increased considerably during this period. Therefore there must be increased phosphorylation of fructose by either hepatic, skeletal or adipose tissues to account for the increased elimination of fructose from the peripheral plasma in piglets from birth to $7 \mathrm{~d}$ of age. The very low fructokinase activity 
found in the intestinal wall of the young pig suggests that fructose was absorbed with little or no metabolism in the intestinal wall (Aherne et al. 1969a).

Although piglets are born with very little subcutaneous fat (Mellor \& Cockburn, 1986), 13.8- and 3.0-fold increases in total body-fat content have been reported to occur from birth to $7 \mathrm{~d}$ and from $7-14 \mathrm{~d}$ of age respectively (Lodge et al. 1978). We calculated $\left(0 \cdot 693 / \mathrm{t}^{1 / 2} \times 100\right)$ that the rates of elimination of fructose from the peripheral plasma were $0.14 \%$ per $\min , 1.82 \%$ per $\min$ and $2.39 \%$ per $\min$, in $2-$, 7 - and 15 -d-old piglets respectively (treatment FRU + GLU). Therefore, the increases in the elimination rate of fructose were 13-0- and 1.4-fold in piglets from 2 to $7 \mathrm{~d}$ and 7 to $15 \mathrm{~d}$ of age respectively. Thus, the increase in the elimination rate of fructose demonstrated in our study was of a similar magnitude to the increase in the deposition of fat in the piglet (Lodge et al. 1978). Even though the affinity of hexokinase for fructose is twenty times lower than its affinity for glucose, the concentration of glucose in adipose tissue is very low (Shafrir, 1985) and thus, in contrast to muscle hexokinase, adipose tissue hexokinase may effectively catalyse the phosphorylation of fructose. Therefore, there is circumstantial evidence to suggest that the effective metabolism of fructose may be partially dependent on the amount of adipose tissue present in the piglet.

We would like to thank Sandra Pember for her excellent technical assistance, Wandalup Farms for the availability of the piglets and their facilities and acknowledge the Pig Research and Development Corporation for granting both a Junior Research Fellowship and the financial support for this project.

\section{REFERENCES}

Aherne, F. X., Hays, V. W., Ewan, R. C. \& Speer, V. C. (1969a). Absorption and utilization of sugars by the baby pig. Journal of Animal Science 29, 444-450.

Aherne, F. X., Hays, V. W., Ewan, R. C. \& Speer, V. C. (1969b). Glucose and fructose in the fetal and newborn pig. Journal of Animal Science 29, 906-911.

Arthur, P. G., Kent, J. C. \& Hartmann, P. E. (1989). Milk lactose, citrate and glucose as markers of lactogenesis in normal and diabetic women. Journal of Pediatric Gastroenterology and Nutrition 9, 488-496.

Atwood, C. S., Toussaint, J. K. \& Hartmann, P. E. (1990). Changes in the composition of milk during let-down in the sow. Proceedings of the Australian Society for Reproductive Biology Annual Meeting, Perth 22, 125.

Bailey, C. B., Kitts, W. D. \& Wood, A. J. (1956). The development of the digestive enzyme system of the pig during its pre-weaning phase of growth. Canadian Journal of Agricultural Science 36, 51-59.

Barber, R. S., Braude, R. \& Mitchell, K. G. (1955). Studies on milk production of Large White pigs. Journal of Agricultural Science 46, 97-118.

Becker, D. E., Ullrey, D. E. \& Terrill, S. W. (1954). A comparison in a synthetic milk diet for the baby pig. Archives of Biochemistry and Biophysics 48, 178-183.

Bird, P. H., Atwood, C. A. \& Hartmann, P. E. (1995). Assessment of lactose digestion in piglets based on responses of blood galactose to oral doses of lactose, galactose plus glucose or milk. British Journal of Nutrition 73, 753-761.

Bird, P. H. \& Hartmann, P. E. (1994). The response in the blood of piglets to oral doses of galactose and glucose and intravenous administration of galactose. British Journal of Nutrition 71, 553-561.

Curtis, S. E., Heidenreich, C. J. \& Foley, C. W. (1966). Carbohydrate assimilation and utilization by newborn pigs. Journal of Animal Science 25, 655-661.

Dahlqvist, A. (1961). Intestinal carbohydrases of a new-born pig. Nature 190, 31-32.

Dollar, A. M., Mitchell, K. G. \& Porter, J. W. G. (1957). Further observations of the utilization of glucose and maltose in the young pig. Proceedings of the Nutrition Society 16, xii.

Fraser, D. (1980). A review of the behavioural mechanism of milk ejection of the domestic pig. Applied Animal Ethology 6, 247-255.

Guppy, M., Sabaratnam, R., Devadason, S. \& Whisson, M. E. (1990). Fructose formation in stored blood. Biochemistry International 21, 210-224.

Hartman, P. A., Hays, V. W., Baker, R. O., Neagle, L. H. \& Catron, D. V. (1961). Digestive enzyme development in the young pig. Journal of Animal Science 20, 114-123.

Hopfer, U. (1987). Membrane transport mechanisms for hexoses and amino acids in the small intestine. In Physiology of the Gastrointestinal Tract, 2nd ed., pp. 1499-1526 [L. R. Johnson, editor]. New York: Raven Press. 
James, P. S., Smith, M. W., Tivey, D. R. \& Wilson, T. J. G. (1987). Epidermal growth factor selectively increases maltase and sucrase activities in neonatal piglet intestine. Journal of Physiology 393, 583-594.

Johnson, S. R. (1949). Comparison of sugars in the purified diet of baby pigs. American Institute of Nutrition $\mathbf{8}$, 387.

Kidder, D. E., Manners, M. J. \& McCrea, M. R. (1963). The digestion of sucrose by the piglet. Research in Veterinary Science 4, 131-144.

Lodge, G. A., Sarkar, N. K. \& Kramer, J. K. G. (1978). Fat deposition and fatty acid composition in the neonatal pig. Journal of Animal Science 47, 497-504.

Manners, M. J. \& Stevens, J. A. (1972). Changes from birth to maturity in the pattern of distribution of lactase and sucrase activity in the mucosa of the small intestine of pigs. British Journal of Nutrition 28, $113-127$.

Mellor, D. J. \& Cockburn, F. (1986). A comparison of energy metabolism in the newborn infant, piglet and lamb. Quarterly Journal of Experimental Physiology 71, 361-379.

Shafrir, E. (1985). Effect of sucrose and fructose on carbohydrate and lipid metabolism and the resulting consequences. In Regulation of Carbohydrate Metabolism II, pp. 95-140 [R. Beitner, editor]. Boca Raton, FL: CRC Press.

Walker, D. M. (1959). The development of the digestive system of the young animal. II. Carbohydrase enzyme development in the young pig. Journal of Agricultural Science 52, 357-363.

Printed in Great Britain 